Revue des patrimoines

\title{
La constitution d'un corpus des peintures murales de la France : entre utopie et réalité
}

Groupe de Recherches sur la Peinture Murale (GRPM)

\section{OpenEdition}

1 Journals

Édition électronique

URL : https://journals.openedition.org/insitu/10859

DOI : $10.4000 /$ insitu. 10859

ISSN : 1630-7305

Éditeur

Ministère de la Culture

Référence électronique

Groupe de Recherches sur la Peinture Murale (GRPM), "La constitution d'un corpus des peintures murales de la France : entre utopie et réalité », In Situ [En ligne], 22 | 2013, mis en ligne le 18 décembre 2013, consulté le 21 septembre 2021. URL : http://journals.openedition.org/insitu/10859 ; DOI : https://doi.org/10.4000/insitu.10859

Ce document a été généré automatiquement le 21 septembre 2021.

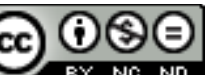

In Situ Revues des patrimoines est mis à disposition selon les termes de la licence Creative Commons Attribution - Pas d'Utilisation Commerciale - Pas de Modification 4.0 International. 


\title{
La constitution d'un corpus des peintures murales de la France : entre utopie et réalité
}

\author{
Groupe de Recherches sur la Peinture Murale (GRPM)
}

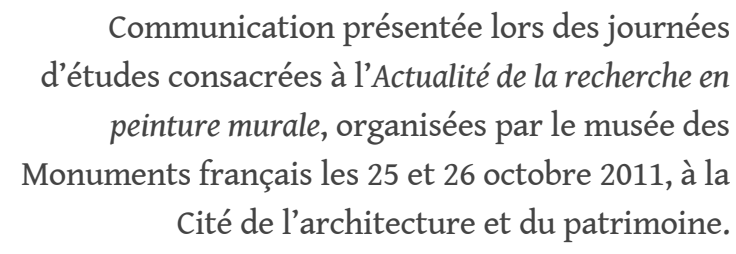

L'idée d'un corpus de la peinture monumentale en France peut paraître utopique au vu de l'ampleur du matériau à étudier, constamment enrichi par de nouvelles mises au jour et par les recherches de terrain ${ }^{1}$. Mais l'importance en nombre et en qualité de ces découvertes, l'ampleur de leur répartition géographique et chronologique, la variété des approches scientifiques et les questions de conservation engendrent de nombreux besoins que l'on pourrait regrouper autour de trois axes - conservation, diffusion (documentation) et valorisation (grand public et recherche) -, témoignant de la nécessité de dépasser d'inévitables difficultés et d'aboutir à l'établissement d'un tel corpus.

De multiples tentatives ont d'ailleurs abouti à des synthèses, au niveau régional et à l'étranger, permettant de dresser un bilan positif, encourageant l'utilisation de nouveaux outils pour la documentation et la recherche dans le domaine de la peinture monumentale. Plusieurs expériences similaires, réalisées sur d'autres corpus d'œuvres (vitrail, manuscrits...) ont montré leur efficience et constituent certainement des préalables à considérer pour la constitution d'un corpus de la peinture monumentale.

3 Les réflexions sur le sujet semblent lancées depuis longtemps, pourtant les difficultés de mises en œuvre sont bien réelles, elles ont constamment repoussé le projet d'un corpus national. Nous proposons ici un état des lieux des expériences engagées, des difficultés identifiées, des choix opérés. Nous voulons aussi proposer des pistes de réflexion pour la constitution d'un nouvel outil coopératif, fédérateur et efficace. 


\section{La notion de corpus : terminologie et significations}

4 Le terme de " corpus ", tel que nous l'entendons ici, peut sans doute être défini comme la forme la plus aboutie et la plus complète d'un inventaire, d'une enquête réunissant un maximum d'informations avec prétention d'exhaustivité. Un bilan historiographique permet de replacer les études - publiées ou non - dans une optique assez large. Chacune d'elles a été menée dans un but spécifique et selon des critères précis. Par conséquent, les termes employés pour désigner ces travaux plus ou moins proches de la notion de corpus ont varié sensiblement. En témoignent les quelques définitions ${ }^{2}$ ci-dessous, employées couramment pour désigner des travaux sur la peinture murale :

5 inventaire: dénombrement d'éléments, groupés par catégories, qui constituent un ensemble cohérent, à un moment donné.

6 - recensement : inventaire, relevé effectué sur un sujet donné dans un domaine donné.

7 - répertoire : inventaire méthodique (énumération, liste, table, etc.) où les informations sont classées dans un ordre qui permet de les retrouver facilement.

8 - bilan : inventaire général des éléments d'une situation, évaluation chiffrée.

9 - enquête : toute recherche, menée dans des secteurs variés en recueillant les réponses et témoignages des personnes ou en rassemblant des documents, donnant lieu à un rapport écrit.

10 - catalogue ou catalogue raisonné : liste, établie dans un ordre donné, de noms de personnes ou de choses formant une collection; la somme connue sur le sujet à un moment donné pour un catalogue raisonné.

11 - base (de données) ${ }^{3}$ : recueil d'œuvres, de données ou d'autres éléments indépendants, disposés de manière systématique ou méthodique et individuellement accessible par des moyens électroniques ou par tout autre moyen.

12 - banque (de données.... ${ }^{4}$ : ensemble de données relatif à un domaine défini des connaissances, généralement organisé et structuré en base de données pour être offert aux utilisateurs. On distingue les banques de données bibliographiques, iconographiques, textuelles, électroniques de type GED, numériques et multimédias.

13 Tous ces termes ont trait à la constitution et au rassemblement de données - de la simple liste à une synthèse thématique - dans le but de fournir un outil, un produit, un ensemble d'informations plus ou moins approfondi, plus ou moins abouti, plus ou moins organisé. La définition du mot "corpus» dans Le Trésor de la langue française fait notamment référence au caractère scientifique et à l'exhaustivité: "Recueil réunissant ou se proposant de réunir, en vue de leur étude scientifique, la totalité des documents disponibles d'un genre donné, par exemple épigraphiques, littéraires, etc. ».

Le corpus procède forcément d'une large enquête des sources et des œuvres conservées et passe obligatoirement par la constitution d'un répertoire issu d'un inventaire ou d'un recensement. Au bout du compte, sa forme pourrait être celle d'un catalogue raisonné avec des notices par site ou celle d'une base de données.

Nous définirions le cadre d'un corpus national des peintures murales par son cadre topographique, correspondant aux limites actuelles de la France, et par son cadre chronologique, couvrant les œuvres réalisées du haut Moyen Âge à nos jours. Ce corpus devrait englober les peintures des édifices religieux et civils, publics et privés, qu'elles 
soient conservées ou disparues mais documentées. Quant à la typologie des décors, le corpus devrait tenir compte des peintures figuratives ou simplement décoratives, de la polychromie architecturale, des décors héraldiques ou encore des traces sous badigeons. Il devrait, pour chaque site, fournir un maximum d'informations, datées, d'ordre historique, historiographique, iconographique, formel, technique, archéologique et sanitaire.

\section{La peinture murale : un corpus qui a pris du retard}

Alors que divers corpus ont été lancés à l'échelle nationale dès les années 1950, celui des peintures murales fait toujours défaut à ce jour. À l'initiative de Louis Grodecki, la série du Corpus vitrearum, créée en 1959, s'est enrichie depuis 1978 de neuf volumes consacrés à des régions (appelés recensements) ${ }^{5}$ et plusieurs autres volumes à caractère monographique consacrés à un édifice particulier ${ }^{6}$. Ces recensements ont été menés au sein du $\mathrm{CNRS}^{7}$. La dynamique de cette recherche sur plus de cinq décennies a conduit à une connaissance approfondie de la presque totalité des vitraux anciens répertoriés en France. Ces études se poursuivent actuellement dans le Sud-Ouest de la France dans les Régions Aquitaine, Midi-Pyrénées et Languedoc-Roussillon.

Une autre entreprise du CNRS, dont les travaux sont également très avancés, concerne les manuscrits. Le corpus des manuscrits datés en écriture latine a été publié entre 1959 et 1985, et constitue depuis un outil incontournable. Celui des manuscrits enluminés conservés dans les bibliothèques de France, lancé en 1979, est, depuis des années, accessible par les bases de données Enluminures ${ }^{8}$ pour les bibliothèques municipales, Liber Floridus $^{9}$ pour les bibliothèques de l'enseignement supérieur et Mandragore ${ }^{10}$ pour la Bibliothèque nationale de France (BnF). À ces outils, il faut ajouter les publications de la BnF sur les manuscrits enluminés conservés dans ses collections, publiés par lieu d'origine. Des programmes de numérisation sont directement liés à ces outils de recherche qui, outre la participation à la constitution d'un corpus des manuscrits enluminés, favorisent leur accessibilité au plus grand nombre et participent à leur conservation.

Citons aussi la vaste entreprise, également menée par le CNRS, du Corpus des inscriptions de la France médiévale, dont vingt-quatre volumes ont été publiés depuis 1974 et couvrent une large partie de la France ${ }^{11}$. Plus récemment mis en place, le Corpus architecturae religiosae europeae, programme international initié en 2002 par l'université de Zagreb, a pour objectif de recenser les édifices religieux antérieurs à l'an Mil. Le programme français a débuté en 2008 avec l'université de Bourgogne et le CNRS, UMR 5594 de Dijon, et c'est à la France qu'est revenue la tâche de mettre au point la base de données informatiques en ligne que tous les pays pourront partager. Enfin, il est à noter aussi les investigations d'associations, comme par exemple Misericordia International qui établit le corpus des stalles médiévales; le volume consacré à la France a paru en $2003^{12}$.

Parmi ces corpus en cours et très avancés, on constate d'emblée le rôle clé du CNRS et des structures qui en découlent et une nette prédilection pour l'époque médiévale.

\section{Les motivations pour la création d'un corpus des peintures murales}

En ce qui concerne la peinture murale, l'absence de corpus national est un cruel constat. Pourtant les tentatives ont été nombreuses depuis le XIX ${ }^{e}$ siècle et les motivations ne 
manquent pas. Celles-ci s'orientent autour de trois axes principaux qui se répondent et se complètent : cette prise de conscience. Paul Deschamps (1888-1974), directeur du Musée de sculpture comparée en 1927, réussit à réhabiliter l'ancien palais du Trocadéro pour l'Exposition universelle de 1937. Dès lors, le nouveau Palais de Chaillot accueillit le «Musée des Monuments français", qui se recentra sur l'Hexagone. La création d'une section consacrée à la peinture murale était prévue dès janvier 1937, mais la première partie, présentant les peintures pré-romanes et romanes, ne fut inaugurée qu'en juin 1945, au premier étage du bâtiment; les deux niveaux supérieurs, consacrés à la peinture gothique, ont été respectivement ouverts en 1954 et 1959. Ces galeries présentaient des copies à l'échelle - réalisées à cette intention et le plus souvent marouflées sur un support architecturé - des principales peintures murales françaises (la voûte de Saint-Savin-surGartempe par exemple ou la crypte de Saint-Aignan-sur-Cher).

Les collaborateurs de Paul Deschamps, notamment Marc Thibout (1905-1991) qui lui a succédé à la tête du Musée en 1959, ont beaucoup œuvré à la réalisation d'un corpus des peintures murales médiévales françaises. Grâce aux tournées dans l'Hexagone et aux notes prises sur le terrain pendant plus de trente ans par Aimée Neury (1909-2006) (fig. $\mathbf{n}$ $\left.{ }^{\circ} \mathbf{1}\right)$, la connaissance a beaucoup progressé en une génération et demie. Les deux ouvrages 
de P. Deschamps et M. Thibout, publiés en $1951^{14}$ et en $1963^{15}$ sur la peinture romane et sur la peinture gothique antérieure à 1380 , reflètent bien le progrès accompli en si peu de temps.

Les publications de cette équipe et les cours de P. Deschamps à l'École du Louvre suscitèrent de nouvelles vocations et attirèrent l'attention des historiens de l'art sur la problématique de la peinture murale. Citons les publications d'Henri Focillon en $1938^{16}$, de Clémence-Paul Duprat en $1942^{17}$, de Paul-Henri Michel en $1949^{18}$ ou les très nombreux articles de Marc Thibout parus dans le Bulletin monumental ou dans le Bulletin de la Société des Antiquaires de France.

Figure 1

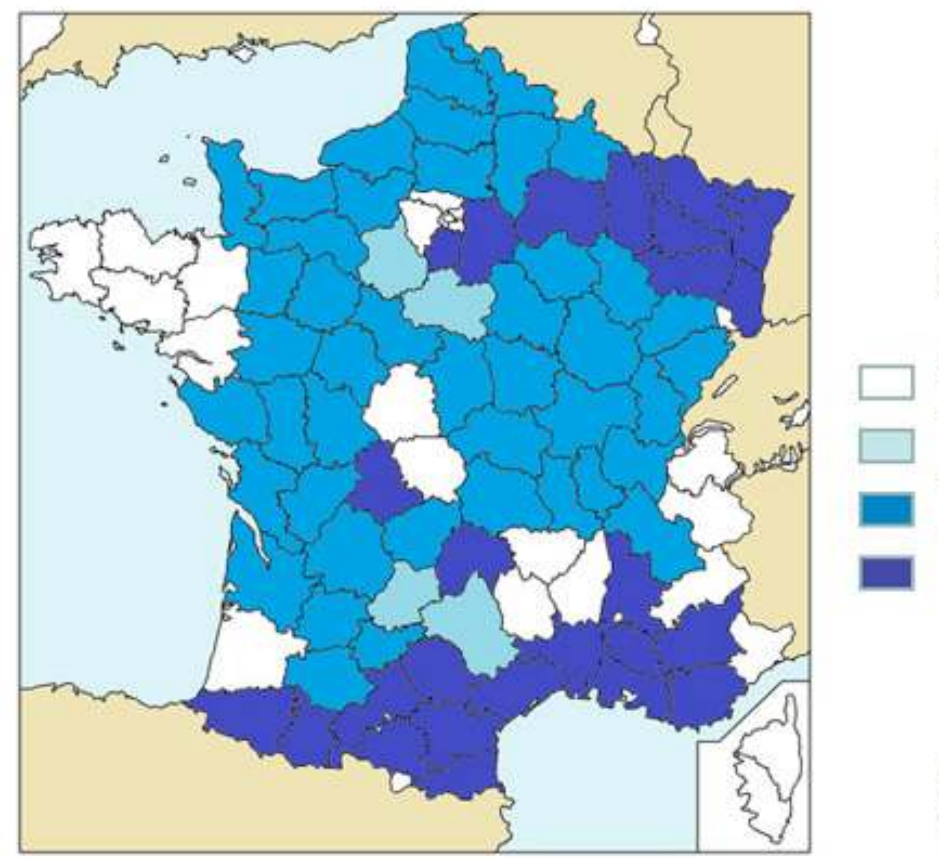

Tournées

d'Aimée NEURY

des années 1940 aux

années 1970

Départ. non prospectés

Tournées des années 1940

Tournées des années 1950

Tournées des années 1960

carte

V. Juhel et I. Hans-Collas

2001

TERRITOIRES COUVERTS PAR A. NEURY AU COURS DE SES PROSPECTIONS DES ANNÉES 1940 AUX ANNÉES 1970.

CARTO. JUHEL, VINCENT, HANS-COLLAS, ILONA, 2001. (C VINCENT JUHEL, ILONA HANS-COLLAS.

Les travaux en régions participèrent aussi largement à cette floraison. On ne peut passer sous silence l'œuvre de Suzanne Trocmé (1887-1971) ou celle de Madeleine Pré (1920-1976), qui ont respectivement travaillé dans le Vendômois et dans le Maine-Anjou pendant toute leur carrière. Leur double casquette d'historiennes de l'art et de restauratrices leur a permis de découvrir, mettre au jour, relever, et surtout publier de très nombreux ensembles, en étroite relation avec le Musée national des Monuments français ou les Monuments historiques.

Marguerite Roques (1884-1973), quant à elle, a inventorié les peintures murales du SudEst de la France dans le cadre de sa thèse, publiée en $1961^{19}$. Comme Suzanne Trocmé et Madeleine Pré, elle a beaucoup travaillé sur le terrain, en privilégiant le dessin et l'aquarelle à la technique photographique. Le Sud-Ouest a été couvert par Robert Mesuret qui a publié en 1967 un riche inventaire des peintures conservées et disparues ${ }^{20}$.

La fin des années 1960 marque cependant le début d'un certain essoufflement, vraisemblablement lié au non renouvellement des générations; pour l'essentiel, les 
travaux publiés furent l'aboutissement de recherches plus anciennes. Le dernier volume de Deschamps et Thibout sur la fin du Moyen Âge ne fut jamais publié et de vastes régions ne sont toujours pas couvertes (moitié Nord de la France). La réalisation du corpus des peintures murales devint plus incertaine.

Le Musée national des Monuments français a toutefois poursuivi son action après la mort de P. Deschamps et de M. Thibout, notamment grâce à ses conservateurs. Ainsi Christian de Mérindol s'intéresse depuis longtemps aux décors monumentaux, auxquels il a consacré un important volume en $2000^{21}$. Son Corpus des décors monumentaux peints et armoriés $d u$ Moyen Âge en France, qui consiste en un recensement documentaire considérable, accompagné de notices descriptives et de références bibliographiques, témoigne tout à la fois de l'importance quantitative des peintures médiévales connues à cette date et de leur grande diversité.

\section{Cadre éditorial}

L'ouvrage d'Otto Demus (1902-1990) consacré à la peinture murale romane en Europe d'abord publié en allemand en 1968, puis en français en 1970 - constitue un autre moment clé, à dimension internationale : l'auteur y posait une nouvelle fois tous les enjeux liés à l'établissement d'un corpus. Ce grand historien d'art autrichien avait bien conscience des problèmes de conservation: "De toutes les formes d'art du Moyen Âge, la peinture murale est celle qui nous est parvenue de la façon la plus fragmentaire "; il précisait que la vaste entreprise d' "un corpus 'complet' aurait rempli de nombreux volumes", insistant aussi sur son impossible exhaustivité: "ce que l'on pourrait considérer aujourd'hui comme complet serait dès demain dépassé. On découvre chaque année de nouveaux cycles ou de nouveaux fragments». Son livre présentait un «choix représentatif » pour « donner au lecteur une juste idée de cet art imposant ${ }^{22}$.

Otto Demus et Otto Pächt occupaient tous deux une chaire à l'Université de Vienne, où ils s'investirent dans la création du corpus des peintures murales médiévales autrichiennes. Dès 1973, ce corpus de grande envergure fut confié à Elga Lanc. La publication du premier volume en $1983^{23}$ fit aussi écho en France. Jean Taralon, alors inspecteur des Monuments historiques, en fit un compte-rendu dans le Bulletin monumenta ${ }^{24}$. Saluant notamment la qualité de l'ouvrage en tant qu'instrument de travail, il exprima aussi le souhait que d'autres pays suivent le modèle autrichien.

En France, les publications de thèses ou de recherches universitaires et institutionnelles, les catalogues d'expositions, mais aussi la publication de travaux de chercheurs locaux se sont multipliés, aboutissant à une couverture presque totale du territoire français; la dimension régionale, départementale et la notion de pays sont privilégiées : Aquitaine, Auvergne, Bourgogne, Lorraine, Midi-Pyrénées, Pays de la Loire, Poitou-Charentes, Indre, Lozère, Manche, Maine-Anjou, Pays niçois, Arc alpin... Mais il est difficile de connaître avec certitude l'état de cette couverture géographique, selon les périodes, la précision des informations, leur actualisation et le degré de traitement (certains secteurs ou édifices font l'objet de plusieurs publications, quand d'autres sont seulement traités une fois ou font seulement l'objet d'une mention). 


\section{Cadre institutionnel}

La base Palissy du ministère de la Culture est la seule base d'envergure nationale qui permette de faire un point à l'échelle de la France. Cette base sur les objets mobiliers livre, pour qui sait l'interroger - et la chose n'est pas toujours aisée -, des résultats sur le nombre de notices de peintures et non sur le nombre de sites à peintures murales en France, ni même sur le nombre de campagnes picturales. En effet, dans la base, un site peut faire l'objet de plusieurs notices et une notice peut renvoyer à plusieurs campagnes picturales; par ailleurs, certaines peintures mentionnées dans des notices de la base Mérimée sont absentes de Palissy. La difficulté de l'interrogation de cette base tient d'une part à l'existence de différents domaines interrogeables (CNMH, ÉTAT, INV, MH, PREDOC). Seuls deux d'entre eux (MH et Inventaire) intéressent notre propos ; l'interrogation peut également se faire simultanément sur les deux domaines. La seconde difficulté tient à un problème de vocabulaire, écueil récurrent dans la constitution des bases de données. En effet l'interrogation avec les termes "peinture murale " et " peinture monumentale ", donne des nombres d'occurrences différents. Il en va de même avec les interrogations du champ OBJET et du champ TEXTE LIBRE. Au début des années 1990, les normes informatiques avaient imposé le choix d'un vocabulaire différent pour la dénomination et la catégorie technique ; les réflexions conduites par Joël Perrin, Christian Davy et MariePasquine Subes ont abouti à retenir "peinture monumentale " pour la dénomination et «peinture murale» pour la catégorie technique. À titre d'exemple, l'interrogation réalisée à l'automne 2011 dans le domaine Inventaire avec le terme "peinture monumentale » dans le champ TEXTE LIBRE - qui permet une recherche dans la totalité des champs de la notice - aboutit aux résultats donnés dans le tableau ci-après (fig. $\mathbf{n}^{\circ} \mathbf{2}$ ). Par ailleurs, une connaissance plus complète du nombre de peintures murales inventoriées en France doit passer par la consultation de la base Mérimée, consacrée à l'architecture. On y trouve les mentions des peintures connues de l'Inventaire. 


\begin{tabular}{|c|c|c|c|}
\hline Colonne1 & Colonne2 & Colonne3 & Colonne4 \\
\hline Les régions françaises & Base PALISSY : peinture murale & Base PALISSY : peinture monumentale & Base régionale \\
\hline Alsace & 21 & 20 & 0 \\
\hline Aquitaine & 41 & 43 & 0 \\
\hline Auvergne & 100 & 97 & 0 \\
\hline Basse-Normandie & 24 & 33 & 0 \\
\hline Bourgogne & 109 & 108 & 0 \\
\hline Bretagne & 25 & 25 & 101 \\
\hline Centre & 99 & 106 & 0 \\
\hline Champagne-Ardenne & 38 & 40 & 0 \\
\hline Corse & 12 & 12 & 0 \\
\hline Franche-Comté & 20 & 25 & 0 \\
\hline Haute-Normandie & 21 & 19 & 0 \\
\hline Ille-de-France & 141 & 145 & 0 \\
\hline Languedoc-Roussillon & 49 & 49 & 0 \\
\hline Limousin & 61 & 48 & 0 \\
\hline Lorraine & 44 & 45 & 0 \\
\hline Midi-Pyrénées & 117 & 115 & 275 \\
\hline Nord-Pas-de-Calais & 10 & 11 & 0 \\
\hline Pays de la Loire & 97 & 95 & 230 \\
\hline Picardie & 49 & 44 & 11 \\
\hline Poitou-Charentes & 81 & 75 & 0 \\
\hline Provence-Alpes-Côte d'Azur & 104 & 96 & 0 \\
\hline Rhône-Alpes & 120 & 115 & 0 \\
\hline TOTAL & 1383 & 1366 & \\
\hline
\end{tabular}

RÉsultats QuANTItATIFs issus d'UNe RECHERCHE « PEINTURE MURALE » ET « PEINTURE MONUMENTALE » DANS LE CHAMP « TEXTE LIBRE » DE LA BASE PALISSY ET INDICATION DU NOMBRE DE Sites À PEINTURES MURALES RÉPERTORIÉs PAR dES ENQUÊTES RÉgIONALES.

TABL. GRPM AUTOMNE 2011. (C) GRPM.

Le domaine MH concerne uniquement les notices des peintures classées Monument historique au titre des objets mobiliers. L'interrogation dans le domaine de l'Inventaire livre donc une moisson numériquement bien plus importante. Cela tient à deux faits. Les notices consacrées aux peintures murales inscrites Monument historique au titre des objets ne sont pas versées dans la base. De même, nombre de protections MH attribuées à des édifices concernent implicitement des peintures murales qui, de fait, n'apparaissent que sous forme de mentions dans les notices. L'évolution de l'appartenance de la peinture murale aux domaines objet ou immeuble par destination complique fortement toute recherche à partir du domaine $\mathrm{MH}$. Cependant il apparaît que le nombre de peintures murales non protégées est beaucoup plus élevé que celui des peintures protégées.

Les résultats de l'interrogation de la base Palissy révèlent une différence de prise en compte des peintures murales selon les territoires, comme en témoigne la carte cidessous (fig. $\mathbf{n}^{\circ} \mathbf{3}$ ). 


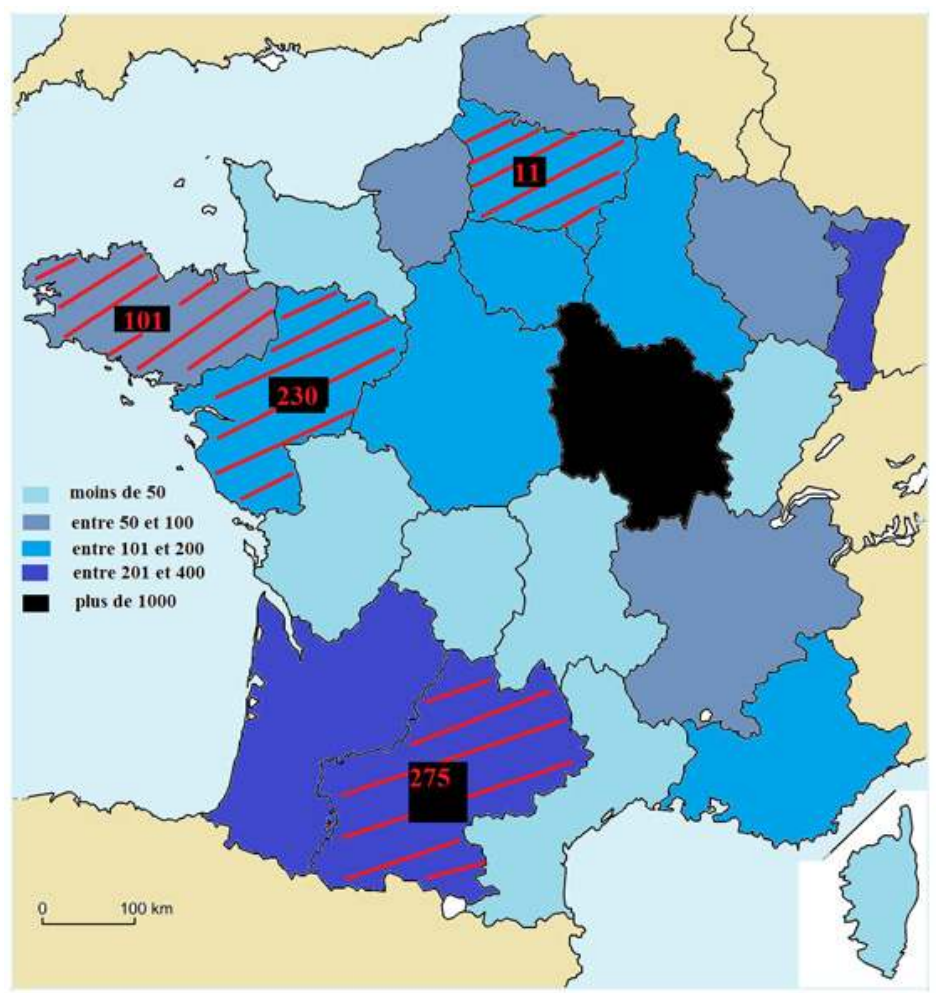

RÉPARTITION QUANTITATIVE ET gÉOgRAPHIQUE DES SITES À PEINTURES MURALES RÉPERTORIÉS DANS LA BASE PALISSY ET PAR DES ENQUÊTES RÉgIONALES.

CARTO. GRPM AUTOMNE 2011. (C) GRPM.

En Bourgogne - à l'instar du succès du Corpus vitrearum - la Région a promu un vaste inventaire des peintures murales, du Moyen Âge à l'époque contemporaine. Réalisé par le Service régional de l'Inventaire, celui-ci aboutit en 1992 à une exposition ${ }^{26}$. Une seconde campagne eut lieu entre 2000 et 2003, conduite cette fois au travers de deux conventions, l'une entre l'Inventaire (DRAC), l'Université et l'association « Patrimoine, ambiances et couleurs de Bourgogne » $(\mathrm{PACOB})$ et la seconde entre $\mathrm{PACoB}$ et la Région. Cette enquête se conclut également par une exposition ${ }^{27}$, associée à un colloque ${ }^{28}$. Un important fonds photographique fut constitué : neuf mille clichés furent réalisés lors du premier inventaire, de 1988-1992, et ce fonds fut doublé lors de la seconde enquête. Depuis 2006, PACoB actualise régulièrement cet inventaire par un travail de terrain, d'étude et de publication $^{29}$.

Depuis l'ouvrage de Robert Mesuret (1967), on connaissait la richesse de la région MidiPyrénées en décors peints monumentaux (peintures murales, plafonds peints, etc.). Depuis 1995, un programme global de connaissance et de sauvegarde de ce patrimoine fragile a été engagé, par le biais d'une enquête scientifique systématique intégrée au 
projet global du SRI et conduite par Sylvie Decottignies, qui renouvelle de manière évidente nos connaissances en la matière dans cette région. Les peintures concernées (époque romane-XVIII siècle) sont systématiquement étudiées; celles postérieures à la période révolutionnaire sont ponctuellement prises en compte en fonction de leur qualité ou de leur iconographie. Cet inventaire thématique consiste en un recensement des œuvres existantes ou subsistantes à l'état de vestiges; les peintures disparues ne sont recensées que si des documents écrits ou figurés précis permettent d'en restituer les dispositions.

41 Chaque peinture recensée fait l'objet d'une notice d'inventaire. Celle-ci peut être minimale ou au contraire la plus complète possible pour les œuvres les plus remarquables qui font l'objet d'une étude scientifique approfondie. Ces notices sont mises en ligne sur la base de données régionale ${ }^{30}$ sous la forme d'un dossier électronique auquel sont liées plusieurs pièces jointes (études, dossiers de restauration, etc.). On accède ainsi à un document multiple élaboré qui présente le résultat du chercheur à une date donnée. Le corpus est régulièrement actualisé en fonction des découvertes et des restaurations. C'est un outil essentiel d'aide à la recherche, permettant d'identifier des problématiques scientifiques, comme la reconnaissance d'une caractéristique régionale, la circulation des artistes et les matériaux employés...

a mise à disposition au plus grand nombre de cet inventaire thématique sur les décors peints monumentaux prend la forme d'un portail Internet de la Région Midi-Pyrénées destiné à mieux transmettre et à mieux valoriser le patrimoine. Cet outil de ressources et de diffusion propose de nombreuses rubriques permettant à l'internaute d'accéder aux inventaires réalisés dans la région, de suivre l'actualité de la recherche et des publications en liaison avec le patrimoine et plus particulièrement à la thématique « décors peints monumentaux ».

Des régions moins réputées pour leurs peintures murales ont toutefois révélé des chiffres non négligeables; ainsi on dénombre en Lorraine plus de deux cents édifices avec des peintures datées entre le $\mathrm{XIII}^{\mathrm{e}}$ et le $\mathrm{XVI}^{\mathrm{e}}$ siècle. En ce qui concerne les régions frontalières, le bilan est également révélateur d'une grande densité. Cinq cent quarante-six sites à peintures murales médiévales ont été repérés dans les Alpes occidentales avec une très forte densité sur l'axe nord-sud du côté français, comme du côté italien ${ }^{31}$.

Pour la région Provence-Alpes-Côte d'Azur, des jalons importants ont été fixés par Marguerite Roques dont l'enquête, dans les années 1960, reste toujours d'actualité et par François Énaud (1920-1992), inspecteur des Monuments historiques, qui donna une réelle impulsion à l'étude de la peinture murale de la région grâce aux nombreuses campagnes de restaurations et à plusieurs publications. En ce qui concerne le département des Hautes-Alpes, une étude d'ensemble a été réalisée et publiée en $1987^{32}$. Il faut également saluer les campagnes photographiques systématiques des peintures médiévales conçues en 1983 par le Centre International de Documentation et de Recherche du Petit Palais d'Avignon ${ }^{33}$ et menées avec la collaboration du ministère de la Culture et des Conseils généraux concernés. Le but était double : conserver et documenter. Des milliers de clichés ont ainsi été réalisés ${ }^{34}$ et divers outils ont été créés pour les rendre accessibles ${ }^{35}$. Ils sont consultables à la photothèque du Centre et un dossier scientifique complet et méthodique a été constitué pour chaque œuvre.

Autre région phare, les Pays de la Loire ont récemment conduit une vaste prospection sous la direction de Christian Davy, en vue notamment d'études approfondies, dont la présentation des résultats est donnée au sein de la présente livraison d'In Situ ${ }^{36}$. 


\section{Cadre universitaire et de la recherche}

En France, les années 1980-1990 ont marqué un tournant. Les recherches menées dans le cadre universitaire révélèrent de nouveaux corpus de peintures murales étudiées à échelle régionale. Parmi elles, de nombreuses thèses de doctorat portant sur le Moyen Âge ont été initiées (fig. $\left.\mathbf{n}^{\circ} \mathbf{4}\right)$ : Michelle Gaborit (Aquitaine), Anne Courtillé (Auvergne du Moyen Âge), Annie Regond (Auvergne du XVI ${ }^{e}$ siècle), Marie-Gabrielle Caffin (Bourgogne), Ilona Hans-Collas (Lorraine), Christine Leduc (Maine et Anjou), Vincent Juhel (Normandie), Christian Davy (Pays de la Loire), Claudine Landry (Poitou) et Virginie Czerniak (Quercy).

Figure 4

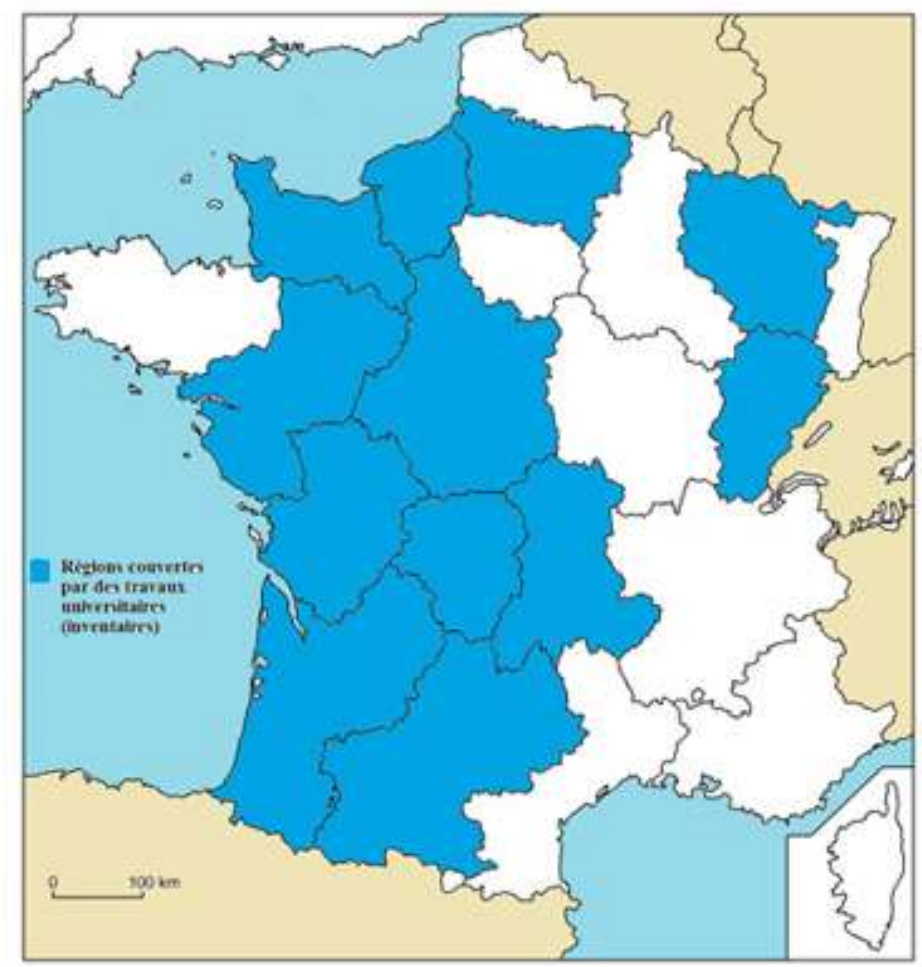

TERRITOIRES COUVERTS PAR LES RECHERCHES DOCTORALES CONDUITES DANS LES ANNÉES 1980-1990. CARTO. GRPM 2011. (C) GRPM.

Le perfectionnement des outils de recherche sur l'Internet permet une plus large visibilité des thèses françaises récentes, en préparation ou soutenues. Une recherche, effectuée à l'automne 2011, par mots-clés ("peinture murale», "peinture monumentale ", « décor peint ») dans Theses.fr ${ }^{37}$ indique seize thèses en préparation en France depuis 2001, dont quatre soutenues depuis 2009 ou sur le point de l'être, tandis que trois ont été abandonnées. Signe encourageant, six des thèses en préparation, soit environ le tiers, viennent de débuter depuis $2010^{38}$. Enfin, signalons au moins une thèse de physique appliquée, soutenue en 2010 à Bordeaux, sur le traitement de la dorure dans la peinture au Moyen $\hat{A g e}{ }^{39}$.

Toutes les thèses mentionnées ici portent sur des peintures murales françaises conservées ou disparues et documentées. La constitution d'un corpus (recensement, 
récolement et collation de nouveaux sites) ne semble plus systématique aujourd'hui. C'est un signe de l'évolution du cadre de la recherche doctorale, imposant des contraintes administratives nouvelles qui interdisent de fait des recherches d'envergure équivalentes à celles préparées dans les années 1990 et démotive de facto les velléités de constitution d'un corpus systématique. Cette tendance est accentuée par la défaveur actuelle d'une partie du monde universitaire pour la constitution de corpus et de ce que l'on nomme avec mépris des thèses d'inventaire : appellation sensée faire référence à des thèses qui se limiteraient à la phase de recensement, excluant une réelle synthèse. En l'absence d'un corpus cohérent et actualisé sur la peinture murale, comment faire autrement que d'établir son propre corpus?

La lecture des sujets montre des approches variées: iconographie, conservationrestauration, pratiques institutionnelles, archéologie, anthropologie, approche formelle et approche technique. Si les thématiques religieuses occupent une place prépondérante, on perçoit un intérêt croissant pour les questions liées aux espaces civils et à leur usage. Les territoires couverts sont vastes et se chevauchent parfois: outre les études d'envergure nationale, on trouve des approches régionales (Alsace, Aquitaine, LanguedocRoussillon, Lorraine, Picardie, Poitou-Charentes, Provence) et une prédilection pour les territoires "anciens" ou culturels, religieux ou laïcs (domaine Plantagenêt, HauteAuvergne, Pays basque, diocèse d'Autun, diocèses de Clermont, du Puy et de Saint-Flour, diocèses de Gap et d'Embrun), mais aussi des zones d'études ignorées, comme la Bretagne. Les périodes traitées sont, elles aussi, variées, puisque le champ chronologique s'étend du $\mathrm{IX}^{\mathrm{e}}$ siècle au $\mathrm{XX}^{\mathrm{e}}$ siècle, avec toutefois une préférence pour le Moyen Âge, dont une proportion importante des études concerne la période allant du XIII ${ }^{e}$ au XVI ${ }^{e}$ siècle.

Mais l'Université a aussi produit de nombreux mémoires de maîtrise et de master, au sujet desquels il est extrêmement difficile d'établir un état précis de la production. Les laboratoires de recherche et les bibliothèques universitaires se réservent en effet le droit de restreindre la diffusion de ces travaux dans le but de protéger leurs auteurs d'éventuels plagiats. Une recherche sur les sites Internet des Services Communs de Documentation $(\mathrm{SCD})$ des différentes universités permet toutefois d'avoir une idée partielle de cette production. À titre d'exemple, examinons le don effectué en 2005 par les héritiers de Michelle Gaborit au bénéfice de la Bibliothèque de l'université de Lettres de Bordeaux 3 : onze mémoires soutenus sous sa direction (officielle ou effective) entre 1992 et 2005 concernent explicitement la peinture murale. Il faut en ajouter au moins cinq autres avec certitude, qui sont absents de ce don, et probablement un peu plus encore, pour une zone d'étude se concentrant sur une grande partie de l'Aquitaine, mais touchant aussi parfois des régions voisines (Poitou-Charentes et Midi-Pyrénées). On pourrait multiplier les exemples en France pour toutes les universités, centres de recherches, laboratoires, directions de recherches...

Médiévistes, modernistes et contemporanéistes ont certainement à s'inspirer des travaux conduits par les chercheurs antiquisants. Citons simplement la composante "peinture murale » de l'UMR 8546 CNRS-ENS «Archéologies d'Orient et d'Occident et textes anciens » (AOROC), en charge de l'étude du décor (peinture et stuc) dans le bâti antique, en Gaule et dans les autres provinces de l'empire romain, de l'époque hellénistique à l'époque omeyyade. L'approche tant matérielle (vestiges archéologiques) qu'historique (sources textuelles) induit une recherche de terrain et en laboratoire qui a pour vocation de produire des outils scientifiques et de diffusion des connaissances. Citons simplement deux projets de cette composante, en relation avec l'objet de nos réflexions, à commencer 
par la base DECORS ANTIQUES, dont l'initiative revient à Alix Barbet, dans le but de concentrer, conserver et transmettre progressivement à la communauté scientifique son fonds d'archives, constitué depuis 1963. Derrière l'appellation générique «décors antiques » sont essentiellement visées les peintures murales hellénistiques, romaines et paléochrétiennes, auxquelles s'ajoutent les mosaïques et les stucs, de l'aire culturelle couverte par la composante "peinture murale ». Une première phase des travaux, soutenue par le Très Grand Équipement (TGE) ADONIS a été livrée début $2009^{40}$ et concerne près de mille trois cents documents, sur la Gaule, librement consultables. Le second projet, DECOR IN SITU, a pour objet la recherche d'une iconographie (clichés photographiques, gouaches, gravures) témoignant de l'état d'un décor et de son environnement lors de sa découverte. Limité au territoire national, il se propose de combler une lacune documentaire, que l'on rencontre également pour les périodes plus récentes, en associant les données concernant les sols décorés et les aménagements (banquette, cheminée, niche, etc.) trouvés à proximité. La prise en considération de ces indices d'occupation, de fonctionnement et d'usure qu'enregistrent les enduits, peints ou non, a pour objectif d'enquêter sur un état de destruction lors des derniers instants d'existence d'un espace.

Tables rondes, brochures et actualisation du site Internet de la composante " peinture murale » à travers plusieurs thématiques de recherche (ex.: «Peintures en place et ensembles fragmentaires $»^{41}$, «Peinture murale et cheminée murale $»^{42}$ ) participent à la diffusion de l'avancée des recherches vers la communauté scientifique. La cartographie des découvertes est actualisée. Une base de données est à l'étude.

Concernant le Moyen Âge, le Centre d'Études Supérieures de Civilisation Médiévale (CESCM) de Poitiers travaille depuis la fin des années 1980 à la constitution d'un corpus des peintures murales de la France, ayant pour vocation de couvrir l'ensemble de la production pour les époques préromane et romane (VIII - XIII ${ }^{\mathrm{e}}$ siècles). Depuis sa création, il s'est accompagné d'une couverture photographique importante et de la création de la base Romane. Sous la direction de Marcello Angheben (responsable de l'équipe " peintures murales ») et d'Aurélia Bolot (responsable de la photothèque), la base originelle a subi une refonte complète dont la dernière mouture est en ligne ${ }^{43}$.

En dépit des objectifs affichés par l'équipe d'étudier pour ce corpus «des peintures murales romanes de la France intégrant l'ensemble des vestiges, des plus modestes aux plus prestigieux $»^{44}$, les campagnes d'intervention ont touché prioritairement les départements situés au Nord des Pyrénées (Aude et Ariège), héritage du travail de John Ottaway, le Poitou, la Touraine et les Pays de la Loire, favorisés pour leur proximité. Les sites privilégiés ont été ceux alliant de préférence à l'intérêt pictural, un intérêt architectural, archéologique, épigraphique...

55 La base documentaire et de recherche Romane constitue le volet de mise à disposition du corpus auprès des publics. Dans un premier temps, elle se limite aux sites majeurs poitevins et des Pays de la Loire. Ce choix peut présenter le risque de maintenir un temps dans l'ombre des sites jugés secondaires ou mineurs, quand la pratique du terrain nous apprend que ces derniers renferment parfois des ensembles peints importants par leur superficie conservée et pour leur intérêt historique. Par ailleurs, la conservation de ces décors est souvent plus incertaine que celle des décors jugés majeurs, de sorte que l'on ignore s'ils seront encore visibles dans quelques années. Enfin, les études sur ces décors « mineurs » manquent cruellement. Ces craintes viennent de l'observation du traitement qui est accordé en général aux peintures «conservées » en contexte civil - plus encore 
dans les édifices non protégés au titre des Monuments historiques - peintures rares, il est vrai, pour la majeure partie de la période couverte par ce corpus.

L'orientation de Romane est largement iconographique : un projet de reconnaissance des formes par l'image est en cours et, en collaboration avec le Groupe d'Anthropologie historique de l'Occident médiéval (GAHOM), l'équipe " peinture murale » a travaillé à la constitution d'un mode de recherche consacré spécifiquement aux problématiques iconographiques de la peinture murale. La version actualisée du Thesaurus des Images Médiévales (TIM) est accessible en ligne, sous l'appellation de TIMEL (Thesaurus des Images Médiévales En Ligne) $)^{45}$.

L'interface de la base a été voulue efficace tout en restant simple d'utilisation. Les fiches sont structurées autour de sept blocs (topographie, architecture, datation, manuscrit/ mobilier/objet, iconographie, épigraphie, description et remarques). Un bloc supplémentaire accueille, pour chaque fiche, plusieurs images issues de la photothèque du CESCM, que l'utilisateur peut exporter avec un choix d'informations extraites de la fiche d'indexation. La longue durée du travail de couverture photographique de même que la pluralité des auteurs permettent de suivre la vie de certaines peintures depuis une cinquantaine d'années et ainsi de conserver la mémoire de certains états, voire de visualiser des clichés d'éléments aujourd'hui disparus ou détruits. On regrettera néanmoins l'absence d'une documentation autre que photographique (sources, bibliographie, données archéologiques, etc.), il est vrai difficile à maintenir actualisée.

Un programme de recherche, européen cette fois, fut initié en 1992 par Dominique Rigaux au sein du CNRS, sous le nom de PREALP ${ }^{46}$. Consacré au recensement et à l'étude des peintures murales des régions alpines (englobant la France, l'Italie et la Suisse), ce vaste corpus visait initialement à inventorier quelques deux cent mille peintures murales du $\mathrm{XIII}^{\mathrm{e}}$ au XVI ${ }^{\mathrm{e}}$ siècle, religieuses et profanes, et à constituer une base de données iconographique exhaustive. "Il s'agissait de produire un instrument de travail permettant d'exploiter tant du point de vue historique qu'artistique l'extraordinaire richesse documentaire que constituent ces œuvres $»^{47}$. Une telle entreprise nécessitait une méthodologie adaptée à la spécificité de la peinture murale ${ }^{48}$. En vingt ans ce projet très ambitieux a malheureusement peu progressé ; un tel chantier devait inévitablement se heurter à nombre de problèmes d'ordre financier, technique, pratique mais aussi intellectuel et scientifique. À ce jour, seule une petite partie est réalisée et un nombre relativement restreint de fiches est accessibles en ligne ${ }^{49}$.

\section{Cadre associatif}

59 Les actions conduites localement ou dans un cadre associatif visant à l'inventaire et à la valorisation d'un patrimoine mal connu, sont également nombreuses. À titre d'exemple, on peut citer les investigations de Pascal Fournigault ${ }^{50}$ en Dordogne, à la fin des années 1980. Ce travail, qui avait bénéficié de financements publics (DRAC), portait sur une période large (Moyen Âge - $\mathrm{XX}^{\mathrm{e}}$ siècle), tant sur les édifices religieux que civils, intégrant notamment des peintures extérieures du XXe siècle (façades en trompe-l'œil par exemple). Issu d'un inventaire des sites documentés et d'une recherche sur le terrain, il se compose de fiches signalétiques très courtes (une page en général) contenant les informations essentielles (localisation, description, proposition de datation, sources et bibliographie succincte). Cet inventaire n'est évidemment plus à jour depuis sa réalisation. 


\section{Bilan des tentatives : défauts et qualités} constitution d'un corpus national est évidente. L'ensemble de la filière institutionnelle en charge de la recherche (Université, CNRS, Grandes Écoles, Services régionaux de l'Archéologie et de l'Inventaire), de la protection (MH) et de la valorisation (musées, au premier rang desquels le Musée national des Monuments français) des peintures murales est concerné. Mais depuis une vingtaine d'années l'engagement de quelques Régions a certainement constitué le principal apport en la matière, par l'acquisition de données, parfois régulièrement actualisées, sur la production régionale; ces initiatives ont notamment permis de faire croître considérablement le nombre d'ensembles peints connus. Pour autant, la cohérence d'un corpus national pâtit de cette situation, chacune des composantes investies dans sa constitution optant pour des critères d'enregistrement des données et de valorisation qui lui sont propres. Ainsi, la visibilité et l'efficacité cumulative potentielle de ces actions s'en trouvent amoindries et ce, malgré l'emploi de plus en plus fréquent d'Internet et de technologies favorisant l'interopérabilité, notamment pour la phase de valorisation. En d'autres termes, on peut regretter que les efforts de plus en plus sensibles en faveur du recensement, de la conservation, de la documentation, de la connaissance et de la valorisation des peintures murales ne 
convergent pas vers une action commune. En toute logique, il faudrait souhaiter une organisation qui soit à même d'empêcher qu'elles se parasitent en quelque sorte par leur dispersion.

En ce qui regarde les périodes chronologiques valorisées, il semble, toutes proportions gardées, que le Moyen Âge ait la faveur des promoteurs de ces actions de recensement. Toutefois, il suffit de revenir sur le travail effectué en Bourgogne, en Midi-Pyrénées, en Dordogne et dans les Pays de la Loire, pour s'apercevoir que cette impression est à nuancer. En effet, ces trois régions et ce département, à la pointe en la matière, ont clairement opté ces dernières années pour une couverture diachronique allant du Moyen Âge à l'époque contemporaine.

revanche, on discerne bien une constante dans le choix presque systématique de considérer uniquement les ensembles peints jugés a priori importants ou des édifices dits "majeurs" des territoires concernés. Dans le même temps, les outils de consultation rendent difficile l'étude de ces ensembles peints pour eux-mêmes, ou comparés les uns aux autres, ne serait-ce qu'au sein d'un même édifice. En effet, l'unité privilégiée d'accès à l'information concernant une peinture est le site, l'édifice et non la peinture elle-même, à l'exception notable de l'opération PréAlp qui est plutôt fondée sur les unités iconographiques. Souvent, pour un site, une seule peinture sera ainsi valorisée, qui plus est dans un état donné, ne permettant pas alors de comprendre l'évolution de cette peinture au cours du temps, ni son intégration au sein d'un ensemble visuel, évoluant luimême au gré des campagnes picturales, architecturales, etc.

L'actualisation des données collectées pour chaque ensemble peint n'est pas toujours de mise. On comprend aisément la difficulté d'y parvenir pour des entreprises d'envergure s'établissant sur le long terme - on pense aux longs inventaires publiés en format papier mais quid des corpus bénéficiant d'une publication numérique? Ce point est davantage sujet à caution encore lorsque la variété des données collectées est restreinte, autrement dit lorsque les matériaux fournis au public pour comprendre et se documenter sur les ensembles peints sont réduits à une simple notice comprenant quelques champs d'informations factuelles. Il en va autrement des supports mettant à disposition une iconographie sur le long terme et une documentation riche, issue d'une analyse (téléchargeable par exemple).

Quoi qu'il en soit, les outils de mise à disposition des informations recueillies sont pensés de plus en plus de manière à permettre au plus grand nombre d'y trouver satisfaction. Ce point est primordial et rend complexe la constitution de ces outils. Il vise à valoriser auprès d'un large public des peintures dont les propriétaires, publics, ou privés, ignorent souvent la valeur patrimoniale de ce qu'ils possèdent. On peut espérer que cette sensibilisation permette à l'avenir que davantage de peintures soient conservées et/ou documentées.

De ce bilan, on aurait donc tendance à retenir comme point positif l'intérêt constant depuis un siècle en faveur de la constitution d'un corpus des peintures murales, aspect contrebalancé par le fait que les nombreuses initiatives et la diversité des acteurs - pour engageantes et riches qu'elles soient - témoignent aussi d'une situation problématique. De ce défaut d'impulsion commune résulte en effet une dispersion de l'information, des énergies et des moyens humains, techniques et financiers. De ce fait, les outils et inventaires sous-exploités au regard des motivations exprimées au début de la présente communication (problématiques de conservation, de recherche et de valorisation), peu 
visibles ou difficiles à intégrer dans des spéculations suprarégionales, sont délaissés par les professionnels qui souvent ignorent totalement leur existence.

\section{Expériences récentes de corpus à l'étranger}

70 La comparaison avec d'autres pays européens permet de constater que la mise en place d'un corpus national s'y heurte aussi à des problématiques similaires. Pourtant, les efforts menés dans certains pays sont importants. Au Danemark, Axel Bolvig, professeur à l'Université de Copenhague, a initié la mise en ligne d'une vaste base de données, riche de dix mille cinq cents peintures murales ${ }^{53}$. En Angleterre, Anne Marshall propose un site Internet consacré aux peintures murales des églises paroissiales, conçu comme un catalogue régulièrement enrichi et actualisé ${ }^{54}$. Les peintures murales font l'objet en Suède de publications assez fournies ${ }^{55}$ tandis qu'en Allemagne et en Italie, elles sont de type monographique ou sous forme de corpus régionaux.

71 Il serait hors de propos, et par ailleurs vain, d'espérer recenser ici la totalité des entreprises conduites à l'étranger et de tenter de dresser le paysage des territoires couverts. Deux expériences, de contextes et d'envergures différents, méritent néanmoins d'être citées à titre d'exemples.

En Belgique, l'Institut Royal du Patrimoine Artistique (IRPA) mène un projet d'inventaire des peintures murales médiévales depuis 2009 : il s'agit essentiellement d'un recensement des peintures murales datant de 1300 à 1550, d'après la documentation existante et l'actualisation de celle-ci ${ }^{56}$. L'organisation est fondée sur un travail collectif : missions de prospection, missions photographiques, missions de recherche, notamment en collaboration avec des conservateurs-restaurateurs et des chimistes. Cet inventaire comprend actuellement deux cent soixante-dix édifices avec des décors peints du XIV ${ }^{\mathrm{e}}$ au $\mathrm{XVI}^{\mathrm{e}}$ siècle. Une fiche d'indexation a été mise au point pour rassembler un maximum de données réunies par l'historien de l'art et le conservateur-restaurateur. Le constat d'état permet d'établir des priorités pour d'éventuelles interventions d'urgence destinées à assurer la conservation des peintures. Alors qu'une large partie de la documentation photographique est accessible en ligne, les dossiers et les fiches sont communiqués de manière plus confidentielle.

73 En Autriche, comme on l'a vu plus haut, l'idée du corpus national remonte aux investigations de Walter Frodl, d'Otto Demus et d'Otto Pächt. Depuis 1973, Elga Lanc, collaboratrice scientifique à l'Académie des sciences d'Autriche, a coordonné cette entreprise dont la publication est hébergée par cette même Académie ${ }^{57}$. Elle a été secondée ponctuellement par d'autres historiens de l'art et par des conservateursrestaurateurs ${ }^{58}$. Le premier tome (1983), consacré à Vienne et à la Basse-Autriche, a révélé cent trente-six édifices à peintures murales ${ }^{59}$, alors qu'un inventaire remontant à 1925 n'en dénombrait que vingt-cinq. Le second volume, paru vingt ans plus tard (2002), recense pour la Styrie cent quarante-huit édifices à peintures murales ${ }^{60}$. Le troisième tome, consacré au Burgenland, est à paraître. Ce corpus présente des notices très complètes sur chaque ensemble avec une analyse très détaillée de l'état de conservation, de l'iconographie et du style, ainsi qu'une bibliographie commentée. Toutefois, en quarante ans de recherches, seule la moitié du territoire a été couverte. Cette lenteur parfois reprochée à cette entreprise - s'explique notamment par la faiblesse des effectifs humains impliqués: le projet repose essentiellement sur l'investissement d'une seule personne. 


\section{Pistes et propositions}

Compte tenu des entreprises présentées, il ne paraît finalement pas utopiste d'appeler à la création d'un corpus national ayant pour objet les peintures murales du Moyen Âge à l'époque contemporaine. Cet appel paraît même évident à en juger par les volontés, pour le moment dispersées, de travailler à la conservation, l'étude et la valorisation de ce patrimoine fragile et méconnu de beaucoup.

Pour autant, un chantier d'une telle envergure nécessite certainement de poser au préalable les grands axes et les enjeux. Nous voudrions ici soumettre des pistes de réflexions thématiques qui nous semblent relever de cette phase préparatoire.

Réflexion sur l'outil : à l'heure actuelle et afin de répondre aux enjeux d'accessibilité au plus grand nombre, il semble primordial de divulguer ce corpus par le biais d'une banque de données en ligne. Cela nécessite la construction d'un outil: une base de données, capable d'intégrer et de permettre l'interrogation de données variées, tant du point de vue de leur nature (images, textes, multimédia...), que de leur type (rapports, études, comptes-rendus, relevés, photos, vidéos, restitutions 3D...), de leur origine (histoire de l'art, histoire, archéologie, conservation-restauration, administration, archéométrie...), de leur niveau de contenu (données factuelles, expertises...).

Réflexion sur la fonction du corpus et sa destination (recherche, enseignement, valorisation patrimoniale), afin d'établir la pertinence des rubriques et des contenus (iconographie, forme, archéologie, technique, etc.). La rédaction d'un protocole pour sa constitution (unité minimale, thesaurus...) sera aussi indispensable.

Réflexion sur l'objet même "peinture murale »: vestige archéologique, image isolée, objet patrimonial, etc. ? Peut-être tout cela à la fois ?

Réflexion sur la périodisation et les ensembles à privilégier (hiérarchisation) : à côté des grands ensembles «majeurs» de la production toutes périodes confondues, il serait bon de réfléchir sur la nécessité de l'étude des pans laissés dans l'ombre (périodes peu étudiées, décors des petits édifices, décors des édifices privés, décors réputés trop fréquents et par conséquent peu considérés, polychromie architecturale, décors visibles sous badigeons...).

Réflexion sur les outils techniques à mettre en œuvre et leur pérennité : base de données, logiciel, équipe d'informaticiens et ingénieurs (expertise, construction et maintenance), accessibilité, mise en ligne.

Réflexion sur les droits liés à la documentation iconographique.

Réflexion sur les moyens humains (« indexants», actualisation des données, constitution d'un réseau « peintures murales », réactivité).

Réflexion sur les moyens financiers.

4 Réflexion sur les moyens légaux et administratifs (législation $\mathrm{MH}$ et nouvelle répartition des compétences, prise en compte des peintures murales, présence d'historiens de l'art spécialistes des peintures murales sur les chantiers, etc.).

Réflexion sur la coordination nécessaire à cause de la multiplication des acteurs concernés et la dispersion de la documentation et des données recueillies. Ces acteurs signalent régulièrement des ensembles peints souvent inconnus et favorisent 
l'acquisition de données matérielles, d'autant plus importantes lorsqu'il s'agit de peintures vouées à disparaître.

Parmi les acteurs susceptibles d'intervenir dans cette concertation, il serait souhaitable d'intégrer :

- Les institutionnels tels: le Musée national des Monuments français; les services patrimoniaux des Monuments historiques, des Services Territoriaux de l'Architecture et du Patrimoine (STAP), Services Régionaux d'Archéologie, Services Régionaux de l'Inventaire, Médiathèque de l'Architecture et du Patrimoine ; les équipes appartenant au CNRS, Universités, Grandes Écoles.

- Des acteurs trop souvent négligés: bureaux d'études et chercheurs indépendants (missionnés par les Institutions, les collectivités locales ou les particuliers); les conservateurs-restaurateurs, les musées, le Laboratoire de Recherche des Monuments Historiques (LRMH), le Centre de Recherche et de Restauration des Musées de France (C2RMF), les architectes du Patrimoine et laboratoires de recherches indépendants; les propriétaires: Collectivités territoriales, particuliers et le réseau local des Sociétés savantes, associations, chercheurs.

Enfin, il serait souhaitable que la signature des auteurs apparaisse en bonne place dans chacun des documents du corpus, afin que chacun d'eux conserve son identité au sein de cette entreprise commune, au travers de ses méthodes, de ses thématiques privilégiées, etc.

\section{GRPM}

Le Groupe de Recherches sur la Peinture Murale (GRPM), fondé en 1997, réunit des chercheurs et des conservateurs-restaurateurs dont le but est de mettre en commun leurs connaissances sur la peinture murale et de développer des projets de recherche. Depuis la fondation, les membres du groupe organisent des « Rencontres » annuelles dans une région spécifique (en France et dans des pays limitrophes). Un bulletin de liaison, Murs...murs, à diffusion électronique, leur permet d'échanger les informations concernant la peinture murale.

Le site internet du GRPM (http://www.grpm.asso.fr/) donne un aperçu des activités du groupe, la liste des membres actifs et présente l'actualité autour de la peinture murale (publications, colloques, expositions, etc.).

Parmi les travaux du GRPM : un ouvrage de synthèse sur le thème de la Rencontre des trois morts et des trois vifs dans la peinture murale en France publié en 2001, la participation au numéro thématique sur « les arts de la couleur » de la revue Histoire et images médiévales en 2009 et un rapport sur les peintures murales de la chapelle SaintMichel d'Aiguilhe pour la commune d'Aiguilhe en 2010.

Les membres du GRPM : Clara André, Pierrick Barreau, Laurence Blondaux, MarieGabrielle Caffin, Christian Davy, Sylvie Decottignies, Ilona Hans-Collas, Didier Jugan, Vincent Juhel, Sophie Kovalevsky, Christine Leduc-Gueye, Pascal Ricarrère, Géraldine Victoir et Anne Vuillemard-Jenn. 


\section{NOTES}

1. - Rédacteurs de l'article : Ilona Hans-Collas, Pascal Ricarrère (membres du GRPM).

2. - D’après Le Trésor de la langue française : voir le site : http://atilf.atilf.fr/.

3. - D'après le Vocabulaire de la documentation: voir le site: http://www.adbs.fr/ vocabulaire-de-la-documentation-41820.htm.

4. - D'après le Vocabulaire de la documentation: voir le site: http://www.adbs.fr/ vocabulaire-de-la-documentation-41820.htm.

5. - Corpus vitrearum medii aevi: parmi les régions étudiées figurent Paris et la région parisienne, la Picardie et le Nord-Pas de Calais (t. I, 1978), la région Centre et les Pays de la Loire (t. II, 1981), la Bourgogne, la Franche-Comté et la région Rhône-Alpes (t. III, 1986), la Champagne-Ardenne (t. IV, 1992), la Lorraine et l'Alsace (t. V, 1994), la Haute-Normandie (t. VI, 2001), la Bretagne (t. VII, 2005), la Basse-Normandie (t. VIII, 2006), l'Auvergne et le Limousin (t. IX, 2011) : voir le site : http://www.corpusvitrearum.org.

6. - Pour n'en citer que quelques-uns : Notre-Dame et la Sainte-Chapelle de Paris, SaintOuen de Rouen, la cathédrale de Strasbourg ou encore la basilique de Saint-Nicolas-dePort.

7. - Auparavant la «Cellule vitrail» était au sein de l'unité mixte UMR 22 (CNRSInventaire général), aujourd'hui elle est rattachée au Centre André Chastel : voir le site : http://www.centrechastel.paris-sorbonne.fr.

8. - Voir le site : http://www.enluminures.culture.fr/documentation/enlumine/fr. Voir aussi la nouvelle base Initiale : http://initiale.irht.cnrs.fr/accueil/index.php. Les notices de manuscrits du Catalogue Général des Manuscrits (CGM), incluses aujourd'hui dans le Catalogue collectif de France (CCFr), sont issues de l'informatisation du Catalogue général des manuscrits des bibliothèques publiques de France, publié de 1849 à 1993.

9. - Voir le site : http://liberfloridus.cines.fr.

10. - Voir le site : http://mandragore.bnf.fr.

11. - Corpus établi au Centre d'études supérieures de civilisation médiévale (C.E.S.C.M.) de l'Université de Poitiers : voir le site : http://www.mshs.univ-poitiers.fr/cescm/spip.php? rubrique16.

12. - BLOCK, Elaine C. Corpus of Medieval Misericords: France. Turnhout : Brepols, 2003. D'autres volumes, du même auteur, sont consacrés à l'Espagne (2005) et à la Belgique et aux Pays-Bas (2010). Voir le site: http://www.leedstrinity.ac.uk/departments/english/ misericordia/Pages/default.aspx.

13. - Citons à titre d'exemple, le CD-Rom et le site Internet La peinture murale médiévale dans le Midi de la France. Volume $\mathrm{n}^{\circ} 1$. Alpes méridionales, réalisé sous la direction scientifique du Centre International de Documentation et de Recherche du Petit Palais d'Avignon (Sophie Kovalevsky et Marie-Claude Léonelli). Voir le site: http:// www.culture.gouv.fr/culture/medieval/francais/index.htm.

14. - DESCHAMPS, Paul, THIBOUT, Marc. La peinture murale en France, le haut Moyen Âge et l'époque romane. Paris : éd. d'histoire et art (Ars et Historia), 1951. 
15. - DESCHAMPS, Paul, THIBOUT, Marc. La peinture murale en France au début de l'époque gothique, de Philippe-Auguste à la fin du règne de Charles V (1180-1380). Paris : CNRS, 1963.

16. - FOCILLON, Henri. Peintures romanes des églises de France. Paris : Hartmann, 1938.

17. - DUPRAT, Clémence-Paul. "Enquête sur la peinture murale en France à l'époque romane ». Bulletin monumental, 1942, t. 101, p. 165-223 et 1944, t. 102, p. 5-90, 161-223.

18. - MICHEL, Paul-Henri. Fresques romanes des églises de France. Paris : Chêne, 1949.

19. - ROQUES, Marguerite. Les peintures murales du sud-est de la France, XIIIe au XVIe siècle. Paris : Picard, 1961.

20. - MESURET, Robert. Les peintures du Sud-Ouest de la France du XIe au XVIe siècle, Languedoc, Catalogne septentrionale, Guienne, Gascogne, comté de Foix. Paris : Picard, 1967.

21. - DE MÉRINDOL, Christian. La Maison des Chevaliers de Pont-Saint-Esprit. Tome 2. Les décors peints. Corpus des décors monumentaux peints et armoriés du Moyen Âge en France. Nîmes : Conseil Général du Gard, 2000.

22. - Citations tirées de l'introduction de son livre: DEMUS, Otto. La peinture murale romane. Paris : Flammarion, 1970. Par ailleurs, Otto Demus fut l'éditeur scientifique du corpus des manuscrits enluminés byzantins, paru en allemand (Corpus der byzantinischen Miniaturenhandschriften) en 5 volumes, entre 1977 et 1993.

23. - LANC, Elga. Corpus der mittelalterlichen Wandmalereien Österreichs, Band I, Die mittelalterlichen Wandmalereien in Wien und Niederösterreich. Wien : Verlag der Österreichischen Akademie der Wissenschaften, 1983.

24. - Bulletin Monumental, 1984, t. 142-IV, p. 475-476.

25. - Voir les sites : http://patrimoine.region-bretagne.fr ; http://patrimoines.midipyrenees.fr ; http://www.patrimoine.paysdelaloire.fr ; http://inventaire.picardie.fr.

26. - D'ocre et d'azur: Peintures murales en Bourgogne. Dijon / Paris : Musée archéologique de Dijon / RMN, 1992.

27. - Couleur de temps, fragments d'histoire: peintures murales en Bourgogne, XIIe-XXe siècles, catalogue d'exposition, Dijon, musée archéologique, 21 juin-2 novembre 2003. Talant: PACoB, 2003. L'exposition est itinérante et circule depuis 2003 dans l'ensemble de la Bourgogne.

28. - Colloque organisé par le Service Régional de l'Inventaire, l'association PACoB, l'unité mixte de recherche 5594 de l'Université de Bourgogne et le Centre d'Études Médiévales d'Auxerre ; voir RUSSO, Daniel (éd.). Peintures murales médiévales, XIIe-XVIe siècles : regards comparés, actes du colloque, Dijon, Université de Bourgogne, 15-17 septembre 2003. Dijon : éd. universitaires de Dijon, 2005.

29. - Voir notamment les Cahiers PACoB. Un département est traité par numéro : Nièvre, 2006 ; Yonne, 2007 ; Côte d'Or, 2010 ; Saône-et-Loire, à paraître.

30. - Voir le site $:$ http://patrimoines.midipyrenees.fr/.

31. - JULLIAN, Martine, LEBASCLE, Marie-Christine, VAIR, Christian. «Les peintures murales». Dans JOURDAIN-ANNEQUIN, Colette (dir.). Atlas culturel des Alpes occidentales. De la préhistoire à la fin du Moyen Âge. Paris : Picard, 2004, p. 408-409 (cartographie ERICA (Évolutions, Résistances et Identités des Cultures Alpines)).

32. - Peintures murales des Hautes-Alpes, XVe-XVIe siècles. Cahiers de l'Inventaire, $n^{\circ} 7$. Aix-enProvence : Édisud, 1987.

33. - Le CIDRPPA est spécialisé dans l'étude de la peinture médiévale méditerranéenne.

34. - Le site de Notre-Dame des Fontaines à la Brigue comptabilise à lui seul trois mille clichés.

35. - 1985-1986 : vidéodisque de quatre mille images ; 1995 : cédérom (voir note 12); 1996 : mise en ligne du site Internet consacré aux Alpes méridionales (<lien vers http:// 
www.culture.gouv.fr/culture/medieval/francais/index.htm>); 1999: mise en ligne du programme présentant les chapelles peintes du Palais des Papes d'Avignon (voir le site : http:// www.culture.gouv.fr/culture/palais-des-papes/fr/index.html).

36. - Voir l'article de Christian Davy dans ce numéro: La prospection des peintures murales dans les Pays de la Loire.

37. - Voir le site : http://www.theses.fr/.

38. - Il faut ajouter à cette liste une thèse, vraisemblablement abandonnée, en Angleterre (Londres, Courtauld Inst.) ainsi que quatre thèses soutenues récemment en Angleterre (Londres, Courtauld Inst.) et en Suisse (Genève, Lausanne). Parmi elles citons : VICTOIR, Géraldine, sous la direction de Paul CROSSLEY et David PARK. Gothic Wall Painting in Picardy, ca.1250 - ca.1350. University of London : Courtauld Institute of Art, 2010 ; LE DESCHAULT DE MONREDON, Térence, sous la direction de Jean WIRTH. Les décors peints figuratifs de l'habitat en France avant 1350. Université de Genève, 2012.

39. - MOUNIER, Aurélie, sous la direction de BECHTEL, Françoise. Aurum, argentum et aliae res innumerabiles. Les dorures dans les peintures murales médiévales du Sud-Ouest de la France. Thèse de doctorat, Pessac : Université Michel de Montaigne - Bordeaux 3, U. F. R. Histoire, Histoire de l'Art et Archéologie, 2010.

40. - Voir le site : http://129.199.58.244/fmi/iwp/res/iwp_home.html. Cliquez sur le lien, cochez « Invité » et cliquez sur « Login ».

41. - Voir le site : http://www.archeo.ens.fr/spip.php?article624.

42. - Voir le site : http://www.archeo.ens.fr/spip.php?article625.

43. - Accessible depuis le site Internet du CESCM. Voir le site: http://presnum.mshs.univpoitiers.fr/romane/accueil.aspx/.

44. - Voir le site : http://cescm.labo.univ-poitiers.fr/spip.php?rubrique125\&lang=fr.

45. - Voir le site : http://www.mshs.univ-poitiers.fr/thesaurus/.

46. - RIGAUX, Dominique. « PREALP, une banque de données pour les peintures alpines ». Mélanges de l'École française de Rome, Moyen Âge, t. 106, 1994-1, p. 153-170.

47. - RIGAUX, Dominique, LUTZ, Conrad. «PREALP, une banque de données pour les peintures alpines ». Dans RIGAUX, Dominique, LUTZ, Conrad. Paroles de murs. Peinture murale, littérature et histoire au Moyen Âge. Sprechende Wände. Wandmalerei, Literatur und Geschichte im Mittelalter. Grenoble, 2007, p. 5-8.

48. - LÉONELLI, Marie-Claude. «Indexer des peintures murales ». Mélanges de l'École française de Rome, Moyen Âge, t. 106, 1994-1, p. 171-177.

49. - Pour le public, seules environ 240 fiches sont accessibles en ligne : voir le site : http://prealp.msh-alpes.prd.fr.

50. - FOURNIGAULT, Pascal. Inventaire des peintures murales de la Dordogne. Sireuil Les Eyzies : Association culturelle de Commarque / C. P. I. E. de Sireuil, 1989. Le dossier est consultable à la DRAC Aquitaine.

51. - La peinture murale de la fin du Moyen Âge : enquêtes régionales, Actes du $9^{\mathrm{e}}$ séminaire international d'art mural, 10-12 mars 1999. Saint-Savin : Centre International d'Art Mural, 2000 (Cahier n5).

52. - Depuis le début de l'enquête plusieurs publications papier ont déjà paru : CORBEL, Pierre-Yves. Peintures monumentales en vallée d'Aure, Hautes-Pyrénées. Toulouse : Association pour la promotion du patrimoine en Midi-Pyrénées (Itinéraires du patrimoine, 86), 1995 ; DECOTTIGNIES, Sylvie. Peintures monumentales dans la vallée du Louron. Toulouse / Paris : 
centre régional de documentation du patrimoine / centre national de documentation du patrimoine (Itinéraires du patrimoine, 109), 1996 ; DECOTTIGNIES, Sylvie. Les peintures monumentales du XI ${ }^{e}$ au XVIII ${ }^{e}$ siècle: Ariège. Toulouse : Accord éd. (Images du patrimoine, 231), 2004 ; BRU, Nicolas (dir.). Archives de pierre : les églises du Lot. Milan : Silvana Ed., 2011.

53. - Church Wall paintings in Denmark - Danske Kalkmalerier. Voir le site : http:// www.kalkmalerier.dk.

54. - Medieval Wall Painting in the English Parish Church. Voir le site: http:// www.paintedchurch.org.

55. - Citons la publication issue d'une thèse de doctorat : NILSEN, Anna. Program och funktion i senmedeltida kalkmåleri : kyrkmålningar i Mälarlandskapen och Finland 1400-1534. Stockholm : Kungl. Vitterhets historie och antikvitets akademien, 1986.

56. - Voir un premier bilan : HANS-COLLAS, Ilona, GHISDAL, Marie-Hélène, VAN DIJCK, Linda. "L'inventaire des peintures murales médiévales conservées en Belgique : documentation, recherche et conservation, projet mené à l'Institut royal du patrimoine artistique (Bruxelles) ». Dans Enjeux et pratiques documentaires en conservation-restauration. Perspectives pour la recherche, actes des journées d'études organisées par l'Association des restaurateurs d'art et d'archéologie de formation universitaire (ARAAFU) et le Centre de recherche et de préservation des biens culturels (CRPBC) EA 4100 Histoire culturelle et sociale de l'art - Université Paris 1 Panthéon-Sorbonne, Paris, 14-15 octobre 2010. Paris : ARAAFU (Conservation-restauration des biens culturels, Cahier technique ${ }^{\circ} 19$ ), 2012, p. 49-53.

57. - Voir le site : http://www.oeaw.ac.at/kunst/publikationen/wandmalerei.html.

58. - Notamment Ivo Hammer, Eva-Maria Höhle et Miriam Porta.

59. - Voir note 22.

60. - LANC, Elga. Corpus der mittelalterlichen Wandmalereien Österreichs, Band II, Die mittelalterlichen Wandmalereien in der Steiermark. Wien : Verlag der Österreichischen Akademie der Wissenschaften, 2002, 2 vol.

\section{RÉSUMÉS}

La France est particulièrement riche en peintures murales de toutes périodes, présentes dans tout type d'édifices, et dont certaines ont été étudiées à l'époque contemporaine. Des monographies ou des études à caractère plus général ont déjà été réalisées, mais un véritable corpus national n'a jamais vu le jour. La nécessité et les tentatives de mettre en œuvre un tel corpus sont ici replacées dans une perspective historiographique globale et dans un cadre de recherche, afin de présenter un bilan à l'échelle du pays. La recherche, les motivations et les moyens techniques ont considérablement changé au cours du temps; autant de raisons de proposer de nouvelles pistes de réflexions.

France is particularly rich in the number of wall paintings remaining in all architectural contexts, of which only some have been studied in the last century. Monographs and general studies have highlighted this exceptional treasure, but a proper national corpus has never been 
established. Previous attempts at creating such a corpus would be been reintegrated here within a global historiographic perspective and a research framework that would provide both a "state of the question" and a springboard for the future. The striking evolution of research questions and technical tools in recent decades totally justifies this new approach.

INDEX

Mots-clés : peinture murale, Corpus, France, Moyen Âge, Époque moderne, Époque contemporaine

\section{AUTEUR}

GROUPE DE RECHERCHES SUR LA PEINTURE MURALE (GRPM)

contact@grpm.asso.fr 